\title{
K-Nearest-Neighbours with a Novel Similarity Measure for Intrusion Detection
}

\author{
Zhenghui Ma \\ School of Computer Science \\ The University of Birmingham \\ Edgbaston, B15 2TT \\ Birmingham, UK
}

\author{
Ata Kaban \\ School of Computer Science \\ The University of Birmingham \\ Edgbaston, B15 2TT \\ Birmingham, UK
}

\begin{abstract}
K-Nearest-Neighbours is one of the simplest yet effective classification methods. The core computation behind it is to calculate the distance from a query point to all of its neighbours and to choose the closest one. The Euclidean distance is the most frequent choice, although other distances are sometimes required. This paper explores a simple yet effective similarity definition within Nearest Neighbours for intrusion detection applications. This novel similarity rule is fast to compute and achieves a very satisfactory performance on the intrusion detection benchmark data sets tested.
\end{abstract}

Keywords-nearest neighbours, similarity measure, intrusion detection

\section{INTRODUCTION}

In this paper we use a machine learning approach to design and implement an intrusion detection system. The system will help the computer defend against security risks, while very little computer security knowledge is required. This intrusion detection system is supported by the intelligent machine learning and data mining algorithms rather than the highly sophisticated computer security technologies. It detects the malicious activities using a K Nearest Neighbours (KNN) classification method.

$\mathrm{KNN}$ is a method for classifying objects based on the knowledge learned from the training data. One of the key challenges is to come up with a proper way to calculate the distance between data points that represent network activity logs. This paper will present a new way to compute similarity within KNN that is very simple and efficient to implement, and seems to be very well suited to the sort of problem we are tackling. We achieve state of the art performance on two network intrusion detection datasets.

\section{DATA AND METHODOLOGY}

The training set consists of network connection records, which look like the following:

\footnotetext{
“0, icmp, ecr i, SF, 1032, 0, 0, 0, 0, 0, 0, 0, 0, 0, 0, 0, 0, 0, 0 , 0, 0, 0, 509, 509, 0.00, 0.00,0.00,0.00, 1.00, 0.00, 0.00,
}

$255,255,1.00,0.00,1.00,0.00,0.00,0.00,0.00,0.00, \mathrm{sm}$ urf."

There are 41 features. Before we start working with the data, it is advantageous to perform some feature selection since removing the features that are irrelevant for the classification task at hand will reduce the dimensionality of the dataset. This has advantages, including the following: The processing time increases with the number of features when calculating the distances, so feature selection will reduce computation time. In addition, removing noise will increase the detection accuracy.

However, an obvious question is, which of these features are the most relevant and which are not? In some cases, domain knowledge about the meaning of various features may be used to select and use the most informative ones. For cases when such knowledge is lacking, there is a huge literature of research on feature selection methods [9] to decide in an automated manner which features to use. These methods roughly fall in two categories: filters and wrappers. In our work we decided to use the Information Gain, which is a filter approach, because of its intuitive meaning. The Information Gain of a feature is a measure of the information content of that feature - that is, the expected reduction in entropy caused by partitioning the training set on the values of that feature [2].

\section{A) Similarity definition}

The K-nearest neighbour classifier works based on the distance calculated between the query point and each data points in the training dataset. Then we choose the $\mathrm{K}$ closest points and take a vote of their class labels to decide the label of the query point.

Hence, arguably, the crux in K-nearest neighbour classification is the definition of a distance (or equivalently, a similarity). Feature selection is already part of defining similarity because it determines which features are taken into account in the similarity computations. Now it remains to define in which way to take into account the selected features. 
The most common distance function used in K-nearest neighbour classification is the Euclidean distance. The reason for this is simply that it is the notion of distance we are used to - the distance one would measure with a ruler. However, when it comes to high dimensional complex data types, the Euclidean distance is no longer suitable $[3,8]$ or not applicable at all. Indeed, how could one calculate the distance between two data points such as "udp, https, 2, 20, 1, 9, 21, 2, 9" and “tcp, http, 2, 13, 1, 0, 1, 2, 1, normal”?

To get around this problem, there has been published work in the literature on various distance metrics for mixed features, e.g. [10]. Here we define and use a new and extremely simple way to calculate the similarity between two such data objects, which we describe in the sequel. For two data points $\mathrm{M}$ and $\mathrm{N}$, each of which contains $\mathrm{K}$ features, $\mathrm{f}_{\mathrm{i}}$ will denote the $\mathrm{i}$-th feature. We define the similarity of $\mathrm{M}$ and $\mathrm{N}$ as the sum of 'matches' between their corresponding features. For one feature of $\mathrm{M}$ and the corresponding feature of $\mathrm{N}$, if they are identical or located in the same interval then we define their similarity to be 1 , otherwise the similarity equals 0 . Then we add up all these 1-0 values to get the similarity between these two data points.

Some of the features are categorical, others are integers, and for these it is easy to compare whether they are identical or not. Some other features have continuous values. For these it is not reasonable to only look at whether their values are identical - most of the time they are not identical but still have the same meaning. For example, when the duration for a connection is 9999 seconds in one data point and 9998 seconds in the other, we could say that the durations of these two connections are same. Therefore, for features that take continuous values, we perform a discretisation step before we compute similarity. That is, the actual values are put into several intervals first, and then we apply the idea of similarity by matching the intervals rather than the original values.

Further, some the continuous valued features range from " 0 " to " 1379963888 " for example. If we directly compare two digital values which could range from 0 to 1379963888 , it is very unlikely to find two features with the same value. That means the similarity for the two data points of this particular feature would be almost always 0 . Putting these values into intervals can solve this kind of problem too.

Having established the usefulness of binning the feature values, we need to decide about the number of bins. For example, a feature with values from " 0 " to " 100 ", in order to get a more accurate result, we divide it by 10 and put it into 10 different intervals. The total number of intervals to consider highly depends on the range of the values and the actual meaning of the feature. We can allocate these values into 5 intervals, 10 intervals, 50 intervals, 100 intervals or any other number. If the feature is very sensitive to the changes of the value, then we may put these values in a larger number of intervals and vice versa. There is always a trade-off between too many intervals and too few intervals. Common sense and some initial (highly non-exhaustive) experimentation guided us in this choice. Figure 1 illustrates the pre-processing steps that we described on a concrete example.

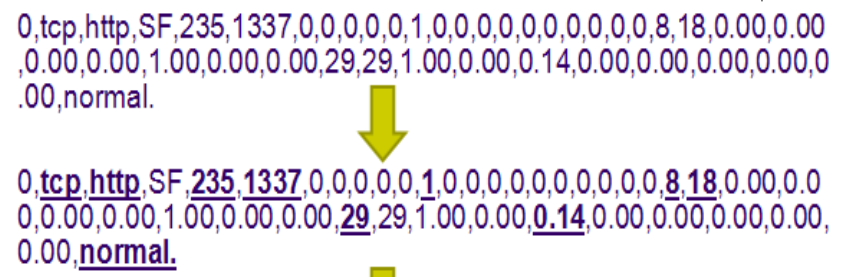

tcp,http, 235,1337, 1,8,18, 29, 0.14,normal.

tcp,http, 2,13,1, 0,1, 2, 1, normal.

\section{Figure 1-Flow-chart of our pre-processing: feature selection and binning.}

Figure 2 then presents the subsequent similarity calculation between two data instances. Notice this is computationally cheap as it only needs to determine whether there is an exact match between a few pairs of values.

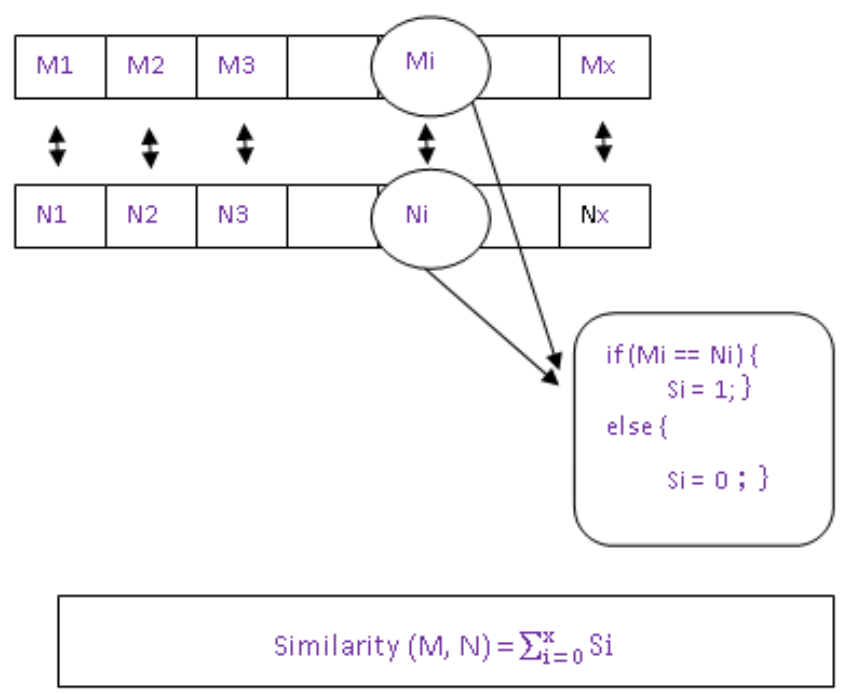

Figure 2- Schematic of our similarity calculation. 
In Figure 2, $\mathrm{M}$ refers to a data point in the training set and $\mathrm{N}$ refers to a query point or a point in the testing set. M1, M2, $\mathrm{N} 1, \mathrm{~N} 2$ and so on refer to features of $\mathrm{N}$, and M respectively. For a concrete example, consider two pre-processed data points "udp, https, 2, 20, 1, 9, 21, 2, 9" and "tcp, http, 2, 13, 1, $0,1,2,1$, normal" - according to our described procedure, their similarity is calculated as the following:

Distance $(M, N)=0+0+1+0+1+0+1+0=3$

\section{B) Choosing the number of nearest neighbours, $K$}

In the K-nearest-neighbour classifier, the class label of the target data point is decided by its closest neighbours. The accuracy of the classification highly depends on the number of nearest neighbours chosen.

Choosing too many neighbours would bring in some noisy data that is irrelevant to the query point, and so the classifier's prediction is an aggregate of all these irrelevant data points. In other words, the classifier under-fits. On the other hand, choosing too few neighbours can also negatively affect the classification accuracy since then the prediction is based on too few data points, and the classifier over-fits. A small value of $\mathrm{K}$ means that noise data could have a higher influence on the result. However, with our similarity definition we find for this kind of problem that choosing $\mathrm{K}$ is much easier. This is because by construction our similarity may be thought of as defining a finite number of concentric circles with different radiuses whose center is the unlabelled query point. The radius of a circle equals to the distance (inverse similarity) between the query point and any of the training points that have the same similarity score. These points sit on the same circle. Figure 3 illustrates this.

Using this definition, the possible values of $\mathrm{K}$ range from 1 to the number of features selected by the system and the similarity scores range from 0 to the maximum of number of features selected and they are all integer values. For a given similarity, it will find a collection of data points which share the same similarity. For the example we gave earlier, the $\mathrm{K}$ could be 1, 2, 3, 4 or 5 and the distance could be $0,1,2,3,4$, or 5 .

Consequently, we find when setting $K=1$, there is a whole collection of neighbours that have the same largest similarity with the query data point. In fact, $\mathrm{K}=1$ turns out to be enough. In our experience, increasing $\mathrm{K}$ will very quickly result in a huge collection of neighbours, which does not help any further. Therefore, we found that 1 is the best value for $\mathrm{K}$ in this task.

\section{RESULTS}

We test our approach on two different datasets. The first one is the KDD Cup 99 dataset [4], and the other one is a data set taken from [5,6], which is the 'University of Twente in September 2008' dataset.
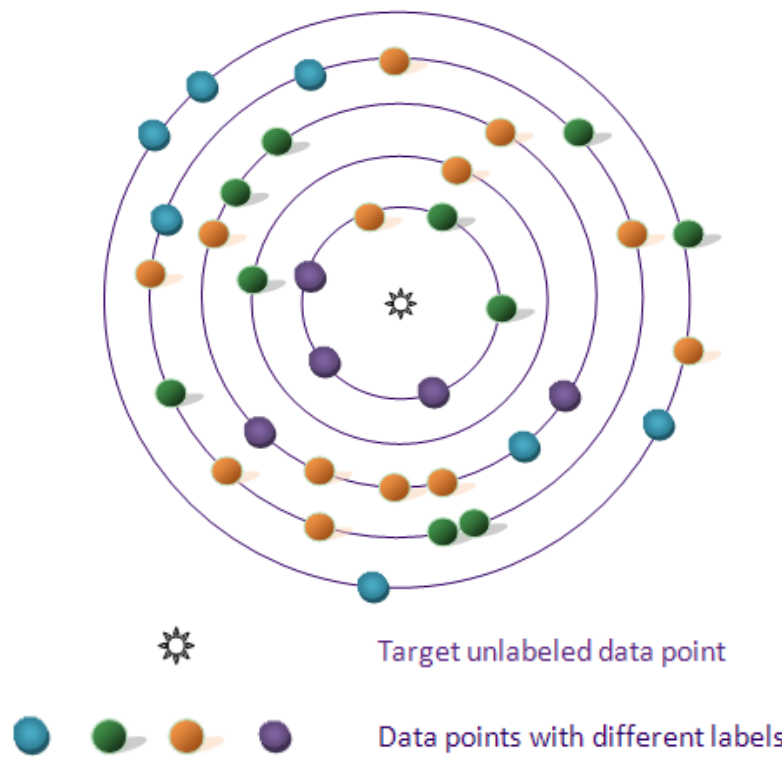

Figure 3 - Concentric circles around the query point (i.e. our target unlabeled point). The training points that sit on the same circle are those that have the same similarity score with the query point.

For the first experimental dataset, we calculate the information gain for the training set and then choose the features with the highest information gain. The number of features selected will affect the accuracy of the classification. Therefore, we experimented with several feature sizes. For the second experimental dataset, we could find more documentation about what the features represented and so our feature selection was based on understanding the actual meaning of these features. E.g. we selected features such as the number of packets in the flow, the number of bits in the flow, the source port number and the destination port number, and ignore the features such as the ID of the connection and the starting time of the connection.

For the testing procedure, we use part of the dataset as the training set and the rest of the data as the testing set. Both the training set and testing set have labels, but the labels of the testing set are not known to the classifier and we use these only to quantify the accuracy of our classifier's predictions. The statistical figures given in the sequel are based on the performance at the end of the testing process. 


\section{A) Detection accuracy.}

Table 1 summarizes the performance of our method on the KDD 99 dataset, when we performed 5 independent repetitions of the experiment, each time creating another random training-testing split. From the table, we can see that the correct detection rate is $90.28 \%$, which means that the system can decide about up to $90 \%$ of the network activities correctly. The system has the false alarm rate of $0.32 \%$, that is, in 32 of 100 cases, the system may generate a false alarm. The missed alarm rate is $6.47 \%$. That is to say, in $6.47 \%$ of the cases the system may fail to generate an alarm when an attack is actually present. In the last line of the table we see that in $1.72 \%$ of the test cases although the system correctly recognises that the network activity is an attack, it fails to recognise the type of the attack correctly.

We also tested our algorithm on another dataset that is both larger and newer, the 'University of Twente in September 2008 ' dataset [6]. The total number of data points contains with in this dataset is about 225,000. Table 2 presents the classification performance on this dataset, not just for five independent splits but also for different proportions of training/testing set sizes. We see our simple algorithm classifies more than $99 \%$ of the test points correctly (except for the final test which has been tested in the extreme splitting proportion of only $0.01 \%$ of points placed in the training set and even then the accuracy is as high as $94.18 \%$.

B) Further analysis of the 'University of Twente in September 2008' dataset.

The highly accurate classification rates were rather surprising, moreover that this newer data set was supposed to be a more realistic one than KDDCup99. It was interesting to inspect the (pre-processed) data in the light of our set of experiments. It turns out that the data has some peculiar characteristics and it is much simpler than one would initially suspect: It contains lots of similar or duplicate data instances. This together with the large size of this dataset means that for most of the testing data points our simple method can almost always find an identical data point from the training set. This of course plays in favour of high classification rates. In addition, there are only 4 categories of class labels and one of these classes contains many more points than the rest. Hence the predicted class label has a higher chance to end up in the majority class. It would be interesting as future work to apply techniques of classification of imbalanced classes.

\section{C) Comparison with previous results on KDDCup99}

The KDD 99 dataset has been used by contestants for intrusion detection competition during the Third International Knowledge Discovery and Data Mining Tools Competition. Several teams of experts were asked to do the same task which is to build a predictive model capable of distinguishing between benign and malicious activities. "The winning entry was submitted by B. Pfahringer [7], and we compare the performance of our algorithm with this winning entry in Figure 4.

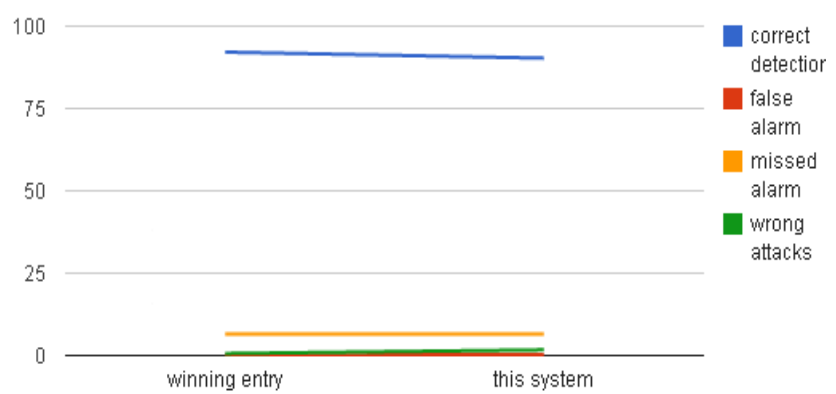

\section{Figure 4-Performance comparison of our approach with the winning entry in [7]. Our simple algorithm performs comparably to the KDDCup999 winner.}

The figure demonstrates that our system is almost as good as the winning entry in the KDD 99 Cup competition. In Figure 4 , we can also see that the correct detection rate is $90.28 \%$ which is just $1.84 \%$ lower than the winning one. The false alarm rate is $0.32 \%$ which is $0.21 \%$ higher than the winning entry. Our method achieves a better missed-alarm rate, which is $0.08 \%$ lower that the winning one. The winning entry achieves a better wrong-attacks rate, which is $1.13 \%$ lower than our system. Generally our approach is comparable with the KDD 99 Cup winner, while our algorithm is extremely simple, easy to implement, and computationally cheap.

\section{CONCLUSIONS}

In this paper we devised a simple yet effective approach to intrusion detection by K-nearest-neighbour employing a novel definition of similarity. As we can see from the experiment results, this novel similarity rule achieves a very satisfactory performance on the data sets tested, and appears to exploit the specifics of this kind of data sets.

The advantage of our similarity rule is that it can handle high dimensional complex datasets easily. In principle, our algorithm is not limited to intrusion detection data sets, and it would be interesting to experiment with it on other application domains in the future. Exploring a possible similarity with some of the ideas in [8] could give us insights about what are the kinds of applications and data sets that our approach is most suited for.

\section{ACKNOWLEDGMENT}

This work was performed as part of the first author's MSc summer project [1] and was generously supported by a Ramsay bursary at the School of Computer Science at Birmingham. 
Table 1-Results on the KDDCup99 data set.

\begin{tabular}{|c||c|c||c|c|c|c||}
\hline Criteria & Dataset 1 & Dataset 2 & Dataset 3 & Dataset 4 & Dataset 5 & Avg \\
\hline \hline correct det & $90.97 \%$ & $90.85 \%$ & $88.06 \%$ & $90.42 \%$ & $91.08 \%$ & $\mathbf{9 0 . 2 8 \%}$ \\
\hline false alarm & $0.00 \%$ & $0.00 \%$ & $0.65 \%$ & $0.64 \%$ & $0.32 \%$ & $\mathbf{0 . 3 2} \%$ \\
\hline missed ala & $7.42 \%$ & $6.62 \%$ & $10.32 \%$ & $6.07 \%$ & $7.96 \%$ & $\mathbf{6 . 4 7 \%}$ \\
\hline wrong att & $1.61 \%$ & $2.52 \%$ & $0.97 \%$ & $2.88 \%$ & $0.64 \%$ & $\mathbf{1 . 7 2 \%}$ \\
\hline
\end{tabular}

Table 2 - Results on the data set 'University of Twente in September 2008'.

\begin{tabular}{|c|c|c|c|c|c|c|c|}
\hline Test\%/Train\% & 1 & 2 & 3 & 4 & 5 & Avg & Std \\
\hline $\begin{array}{c}10-90 \\
(22500 / 202500)\end{array}$ & $100 \%$ & $100 \%$ & $100 \%$ & $100 \%$ & $100 \%$ & $100 \%$ & $\mathbf{0}$ \\
\hline $\begin{array}{c}20-80 \\
(45000 / 180000)\end{array}$ & $100 \%$ & $100 \%$ & $100 \%$ & $100 \%$ & $100 \%$ & $100 \%$ & $\mathbf{0}$ \\
\hline $\begin{array}{c}30-70 \\
(67500 / 157500)\end{array}$ & $100 \%$ & $100 \%$ & $100 \%$ & $99.98 \%$ & $100 \%$ & $99.99 \%$ & 0.008 \\
\hline $\begin{array}{c}40-60 \\
(90000 / 135000)\end{array}$ & $100 \%$ & $100 \%$ & $100 \%$ & $100 \%$ & $100 \%$ & $100 \%$ & $\mathbf{0}$ \\
\hline $\begin{array}{c}50-50 \\
(112500 / 112500)\end{array}$ & $100 \%$ & $100 \%$ & $100 \%$ & $100 \%$ & $100 \%$ & $100 \%$ & $\mathbf{0}$ \\
\hline $\begin{array}{c}60-40 \\
(135000 / 120000)\end{array}$ & $100 \%$ & $99.98 \%$ & $99.97 \%$ & $99.98 \%$ & $100 \%$ & $99.98 \%$ & 0.012 \\
\hline $\begin{array}{c}70-30 \\
(157500 / 67500)\end{array}$ & $99.98 \%$ & $100 \%$ & $99.97 \%$ & $100 \%$ & $100 \%$ & $99.99 \%$ & 0.01265 \\
\hline $\begin{array}{c}80-20 \\
(180000 / 45000)\end{array}$ & $100 \%$ & $99.98 \%$ & $99.98 \%$ & $99.98 \%$ & $99.97 \%$ & $99.98 \%$ & 0.0098 \\
\hline $\begin{array}{c}90-10 \\
(202500 / 22500)\end{array}$ & $99.99 \%$ & $99.98 \%$ & $100 \%$ & $100 \%$ & $100 \%$ & $99.99 \%$ & 0.008 \\
\hline $\begin{array}{c}95-5 \\
(213750 / 11250)\end{array}$ & $100 \%$ & $100 \%$ & $99.96 \%$ & $99.98 \%$ & $99.94 \%$ & $99.98 \%$ & 0.02332 \\
\hline $\begin{array}{c}99-1 \\
(222750 / 2250)\end{array}$ & $99.98 \%$ & $100 \%$ & $100 \%$ & $100 \%$ & $99.96 \%$ & $99.98 \%$ & 0.016 \\
\hline $\begin{array}{l}99.9-0.1 \\
(224775 / 225)\end{array}$ & $99.91 \%$ & $99.98 \%$ & $99.96 \%$ & $99.98 \%$ & $99.89 \%$ & $99.94 \%$ & 0.0372 \\
\hline $\begin{array}{c}\text { 99.99-0.01 } \\
(224978 / 22)\end{array}$ & $91.93 \%$ & $96.95 \%$ & $98.21 \%$ & $95.64 \%$ & $88.16 \%$ & $94.18 \%$ & 3.6701 \\
\hline
\end{tabular}




\section{REFERENCES}

[1] Z. Ma. Machine learning for intrusion detection, MSc Project Report, School of Computer Science, The University of Birmingham, 2012, unpublished.

[2] J. P. Cunningham and S. J. Delany K-nearest neighbor classifiers, unpublished. Available from: University College Dublin Dublin Institute of Technology web site [Accessed: July 19, 2012]: http://www.csi.ucd.ie/UserFiles/publications/UCD-CSI-2007-4.pdf

[3] D. Francois, V. Wertz, M. Verleysen. The concentration of fractional distances. IEEE. Transactions on Knowledge and Data Engineering, 19(7): 873-886, 2007.

[4] S. Hettich, and S.D. Bay (1999), The UCI KDD Archive. KDD Cup 1999 Data. Available from [Accessed: July 16, 2012]: http://kdd.ics.uci.edu/databases/kddcup99/kddcup99.html
[5] A. Sperotto, Dataset for intrusion detection. Available from: http://traces.simpleweb.org/wiki/Labeled_Dataset_for_Intrusion_Detecti on\#Database_Description [Accessed: October 17, 2012].

[6] A. Sperotto, R. Sadre, F. van Vliet, A. Pras, A labeled data set for flowbased intrusion detection. In: IP Operations and Management. Lecture Notes in Computer Science 5843, Berlin, pp. 39-50.

[7] C. Elkan, Results of the KDD'99 classifier learning contest. Available from: School of Computer Science, Carnegie Mellon University web site: http://cseweb.ucsd.edu/users/elkan/clresults.html

[8] A.Shrivastava, and P. Li. Fast Nearest Neighbour Search in high dimensional binary data. Machine Learning and Knowledge Discovery in Databases, Lecture Notes in Computer Science, Vol. 7523, 2012, pp. 474-489.

[9] I. Guyon, and A. Elisseeff. An Introduction to variable and feature selection. Journal of Machine learning Research 3, 2003, pp. 1157-1182.

[10] D.R. Wilson and T.R. Martinez. Improved heterogeneous distance functions. Journal of Artificial Intelligence Research 6, 1997, pp. 1-34. 